\title{
Challenges Associated with Transmission Experiments in the SEM
}

\author{
J. R. Michael ${ }^{1}$
}

1. Sandia National Laboratories, Materials Science and Engineering Center, Albuquerque, NM USA

STEM has been widely utilized for imaging and analysis of thin samples at much higher accelerating voltages (100 kV and up) in STEM/TEM or dedicated STEM instruments and has been studied for many years.[1] Transmission Kikuchi diffraction (TKD) has perhaps driven the most recent interest in low voltage transmission imaging in the SEM (some refer to this as t-SEM) and thin film microanalysis.[2,3] This paper will discuss the advantages of transmission imaging in the SEM along with some of the current challenges of this technique.

The increased resolution of STEM in SEM imaging, microanalysis and TKD over bulk SEM imaging and analysis techniques is well known and has allowed SEM to be applied to many situations where bulk SEM techniques do not provide sufficient resolution. For example, TKD allows orientation maps to be obtained with a step sizes as small as $2 \mathrm{~nm}$, much smaller than can be obtained with standard EBSD. Figure 1a shows the detector arrangement used in this study and Figure 1b and 1c are TKD maps of a two phase region of a thin sample of the Gibeon meteorite that was mapped with a $3 \mathrm{~nm}$ step size. This is about an order of magnitude increase in the spatial resolution of TKD over EBSD. It is apparent from Figure $1 \mathrm{~b}$ and $1 \mathrm{c}$ that TKD only samples the exit surface of the sample.

Combinations of techniques, like TKD and EDS spectrum imaging, are now possible leading to the potential correlation of composition and crystallographic phase, orientation or texture. The large chamber size of the SEM allows more flexibility in detector number and placement than does the sample environment of a typical STEM/TEM instrument. However, the development of analytical STEM in the SEM has lagged the past efforts of the high voltage STEM community in the area of ensuring that the signals collected are limited to the sample under examination and not from adjacent areas of the sample or from the SEM environment. [4]

Figure $2 \mathrm{a}$ is a TKD map from a fine grained austenite/ferrite region obtained at a step size of $5 \mathrm{~nm}$. Figure $2 b$ is the Ni map extracted from a spectrum image that was obtained simultaneously with the TKD map. The Ni map indicates correctly that the austenite phase has an increased Ni composition as compared to the ferrite. The meteorite should contain only $\mathrm{Ni}$ and $\mathrm{Fe}$ at detectable levels. Examination of the spectrum extracted from the maps shows that the largest peak in the spectrum is Gd followed by $\mathrm{Al}$ and $\mathrm{S}$ while the peaks arising from the sample itself are $\mathrm{Ni}$ and $\mathrm{Fe}$. The $\mathrm{Gd}$ and $\mathrm{S}$ peaks must arise from electron interaction with the TKD phosphor screen and the Al may come from the sample holder and the microscope stage or other parts of the SEM sample chamber.

Further developments and applications of STEM in SEM will require efforts to understand the sample region of the SEM so that sources of spurious signals not related to the sample can be identified and then mitigated. [5]

References:

[1] S. Kimoto et al, Transactions of the Second Conference on Electron Microprobe Analysis (1967) abstract number A1968-330735. 
[2] R. R. Keller and R. H. Geiss, J. Microscopy, 245(2012), p. 245

[3] P. W. Trimby, Ultramicroscopy, 120(2012), p. 16.

[4] D. B. Williams, J. I. Goldstein and C. E. Fiori in "Principles of Analytical Electron Microscopy", ed.D.C Joy, A. D. Romig and J. I. Goldstein, (Plenum, New York), p. 123.

[5] Sandia National Laboratories is a multi-mission laboratory managed and operated by Sandia Corporation, a wholly owned subsidiary of Lockheed Martin Corporation, for the U.S. Department of Energy's National Nuclear Security Administration under contract DE-AC04-94AL85000.

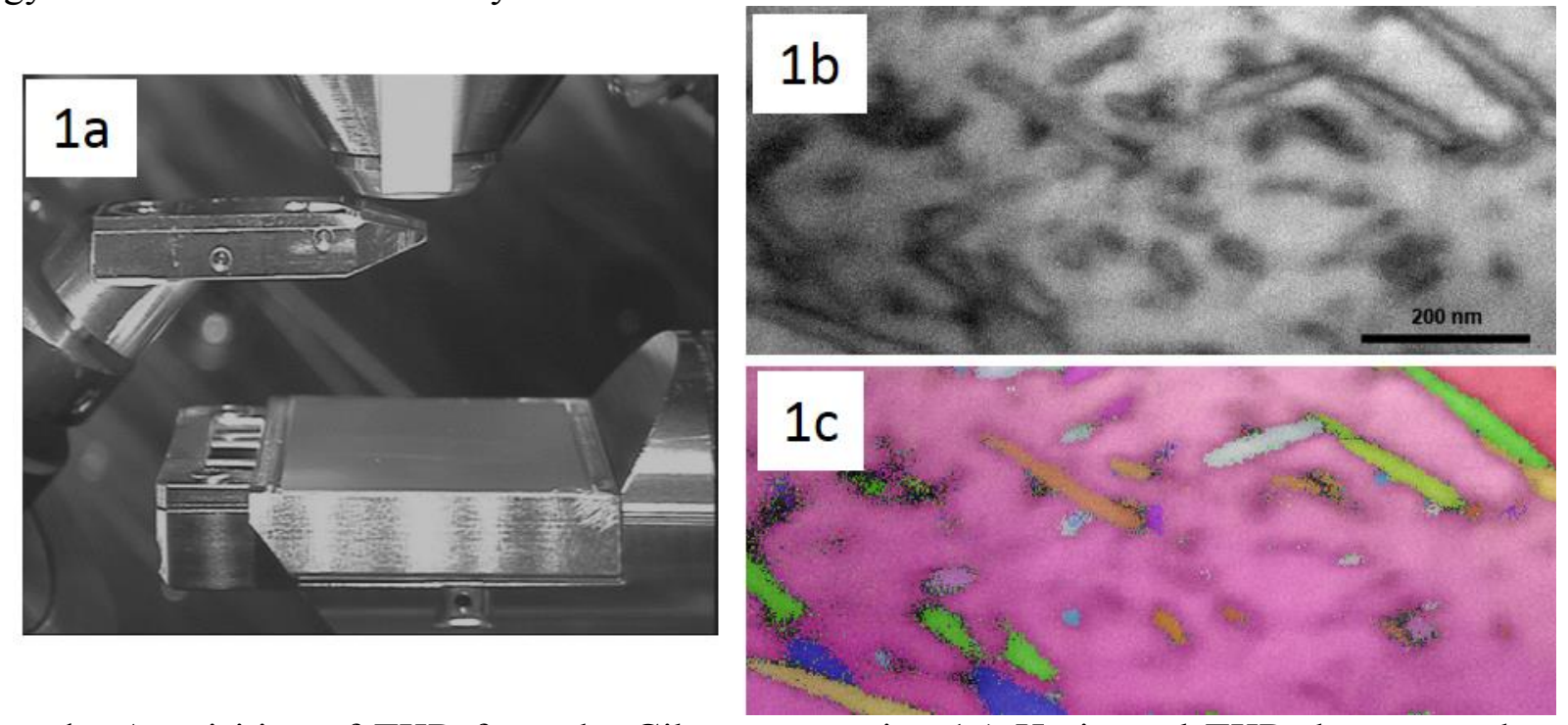

Figure 1. Acquisition of TKD from the Gibeon meteorite. 1a) Horizontal TKD detector and sample holder arrangement, 1b) Band contrast map obtained at $30 \mathrm{kV}$ and $3 \mathrm{~nm}$ pixel spacing, 1c) Inverse pole figure map overlaid on the band contrast map that demonstrates the high spatial resolution of TKD.
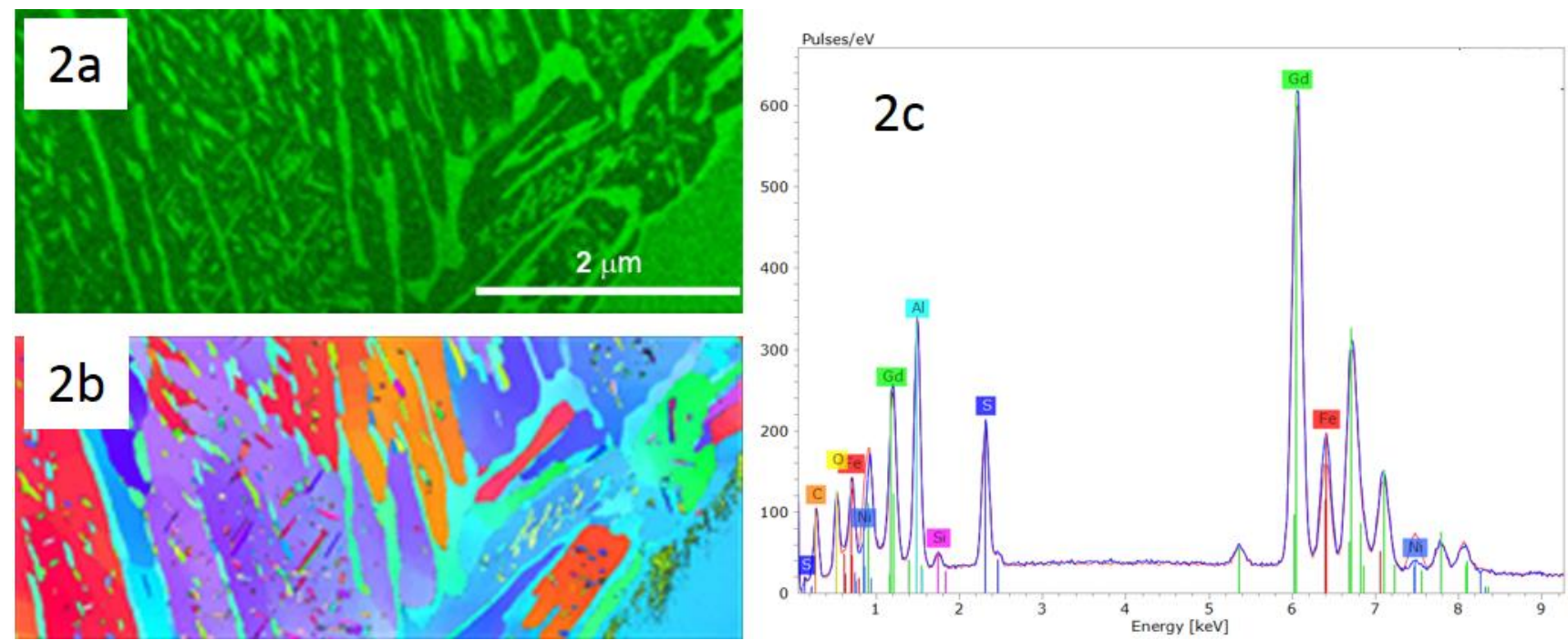

Figure 2. STEM in SEM map results of the Gibeon meteorite acquired at $30 \mathrm{kV}$ with a $5 \mathrm{~nm}$ pixel size. 2a) Ni spectrum image acquired simultaneously with the TKD map shown in 2b. 2c) Summed EDS spectrum from the map shown in $2 \mathrm{a}$. Note that the largest peaks are $\mathrm{Gd}, \mathrm{Al}$ and $\mathrm{S}$, elements not associated with the Gibeon meteorite. 